\title{
Pure endoscopic endonasal odontoidectomy: anatomical study
}

\author{
Andrea Messina • Maria Carmela Bruno • \\ Philippe Decq • Andre Coste • Luigi Maria Cavallo • \\ Enrico de Divittis • Paolo Cappabianca • \\ Manfred Tschabitscher
}

Received: 6 July 2006 /Revised: 8 December 2006 / Accepted: 4 March 2007 / Published online: 10 May 2007

(C) Springer-Verlag 2007

\begin{abstract}
Different disorders may produce irreducible atlanto-axial dislocation with compression of the ventral spinal cord. Among the surgical approaches available for a such condition, the transoral resection of the odontoid process is the most often used. The aim of this anatomical study is to demonstrate the possibility of an anterior cervico-medullary decompression through an endoscopic endonasal approach. Three fresh cadaver heads were used. A modified endonasal endoscopic approach was made in all cases. Endoscopic dissections were performed using a rigid endoscope, $4 \mathrm{~mm}$ in diameter, $18 \mathrm{~cm}$ in length, with 0 degree lenses. Access to the cranio-vertebral junction was possible using a lower trajectory, when compared to that necessary for the sellar region. The choana is entered and the mucosa of the rhinopharynx is dissected and transposed
\end{abstract}

A. Messina $\cdot$ M. C. Bruno - L. M. Cavallo • E. de Divittis •

P. Cappabianca $(\bowtie)$

Department of Neurological Sciences, Division of Neurosurgery,

Università degli Studi di Napoli Federico II,

Via Sergio Pansini, 5,

80131 Naples, Italy

e-mail: paolo.cappabianca@unina.it

P. Decq

Service de Neurochirurgie, Hopital Henri-Mondor, Assistance Publique-Hopitaux de Paris,

Creteil, France

\section{A. Coste}

Service d'ORL et de Chirurgie Cervico-Faciale,

Hopitaux Intercommunal et H. Mondor,

Creteil, France

\section{Tschabitscher}

Center of Anatomy and Cell Biology,

Microsurgical and Endoscopic Anatomy,

Medical University of Vienna,

Vienna, Austria in the oral cavity in order to expose the cranio-vertebral junction and to obtain a mucosal flap useful for the closure. The anterior arch of the atlas and the odontoid process of $\mathrm{C} 2$ are removed, thus exposing the dura mater. The endoscopic endonasal approach could be a valid alternative to the transoral approach for anterior odontoidectomy.

Keywords Endoscopy Odontoid process .

Cranio-vertebral junction

\section{Introduction}

Removal of the odontoid process is a procedure often required for the treatment of the basilar impression with compression of the brain stem or cervical spinal cord due to irreducible atlanto-axial translocation. Different disorders may produce atlanto-axial dislocation such as congenital malformation, chronic inflammation, metabolic disorders and trauma.

The transoral approach is the most favoured approach to the odontoid process and it is largely used for the surgical treatment of extradural and also intradural disorders of the cranio-vertebral junction $[6-9,11-13,15,16,22-26]$. Despite the fact that such an approach provides a direct route to the odontoid process, it presents several disadvantages such as the deepness of the surgical corridor, the sometimes required splitting of the soft palate, the risk of tongue and teeth damage and, in case of dural opening, the increased risk of post-operative CSF leakage and meningitis.

Based on the experience of endoscopic endonasal pituitary surgery $[3,17]$, some works have recently reported anatomical studies and clinical applications of a modified endoscopic endonasal approach for the removal of the dens. These studies show the potential applications of the endoscopic endonasal approach for the surgical manage- 
Fig. 1 a, b Entering the choana, the rhinopharinx and the Eustachian tube have been bilaterally visualized (iwsphs inferior wall of sphenoid sinus, ET Eustachian tube, Rphx rhinopharinx)
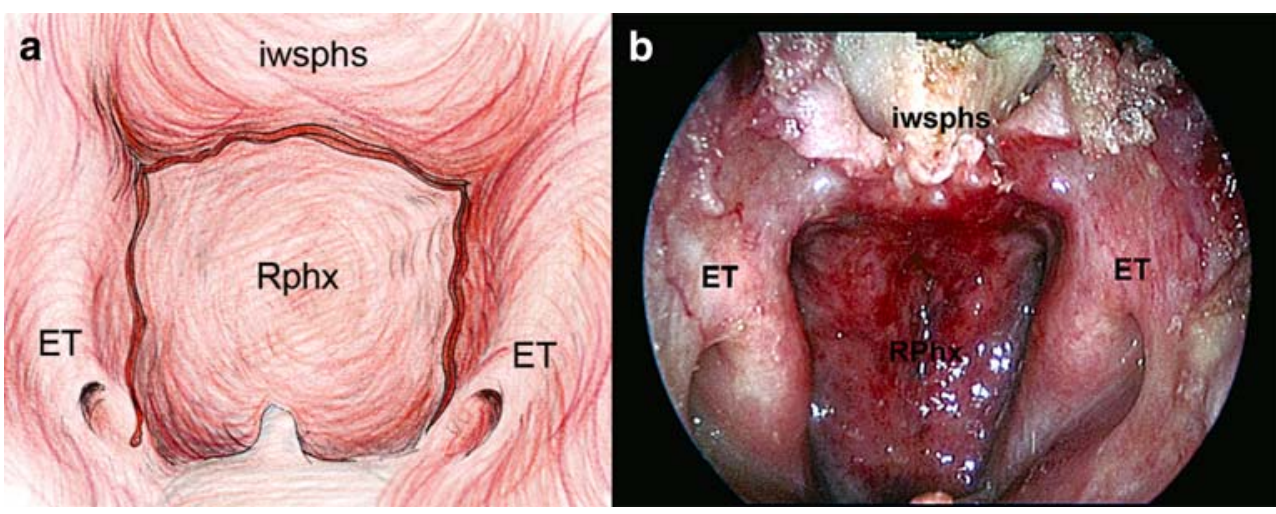

ment of suprasellar, parasellar and retroclival pathologies $[1,2,4,5,10,14,18-21]$.

This anatomic study describes the extended endoscopic endonasal approach to the cranio-vertebral junction, with particular attention to the reconstruction of the surgical route.

\section{Material and methods}

For this anatomic study, three fresh cadaver heads were dissected; an extended endoscopic endonasal approach to the cranio-vertebral junction was performed in all cases.

Endoscopic dissections were performed using a rigid endoscope (Karl Storz GmbH, Tuttlingen, Germany), $4 \mathrm{~mm}$ in diameter, $18 \mathrm{~cm}$ in length, with 0 degree lenses.

The endoscope was connected to a light source through a fiberoptic cable and to a camera fitted with 3CCD sensors. The video-camera was connected to a 21 " monitor supporting the high resolution of the 3CCD technology.

\section{Results}

The procedure started with the introduction of the endoscope into a nasal vestibule through a lower trajectory as compared to the one employed for reaching the sellar region. Along such trajectory, the first structures to be visualized were the nasal septum medially, the inferior turbinate and the middle turbinate laterally. The inferior margin of the middle turbinate led to the choana which represented the main landmark of the approach.

By advancing the endoscope through the choana it was possible to identify the ostium of the Eustachian tube laterally, the rhinopharynx posteriorly, the soft palate inferiorly and the inferior wall of the sphenoid sinus superiorly; the latter representing the superior limit of the surgical approach. Angling the endoscope to the contralateral nasal cavity, it was possible to visualize the ostium of the contralateral Eustachian tube. The ostia of the two Eustachian tubes represented the lateral limits of this approach (see Fig. 1a,b).

In order to expose the cranio-vertebral junction the mucosa of rhinopharynx was incised along its lateral limits at the edge with the ostia of the Eustachian tube and along the inferior wall of the sphenoid sinus superiorly (see Fig. 2a). The mucosa, the longus capitis and longus colli muscles were gently dissected downward as a single layer, thus creating a muscle-mucosal flap (see Figs. $2 b$ and 3). Proceeding from the inferior wall of the sphenoid sinus to the soft palate of the lower clivus, the atlanto-occipital membrane, the anterior arch of $\mathrm{C} 1$ and the body of $\mathrm{C} 2$ were visualized.

Introducing the endoscope in the oral cavity it was possible to reach the dissected mucosa of rhinopharynx and to transpose the muscle-mucosal flap into the oral cavity (see Fig. 4a,b).

This manoeuver permitted an adequate endonasal exposure of the cranio-vertebral junction without removing the
Fig. 2 a The mucosa of the rhinopharynx has been incised in order to create a mucosal flap. b The muscles longus capitis and colli have been dissected together with the mucosa in order to expose the craniovertebral junction. (ET Eustachian tube, Rphx rhinopharinx, NS nasal septum, iwsphs inferior wall of sphenoid sinus, $C$ clivus, aom atlantooccipital membrane, $C 1$ atlas, $m m f$ muscle-mucosal flap)

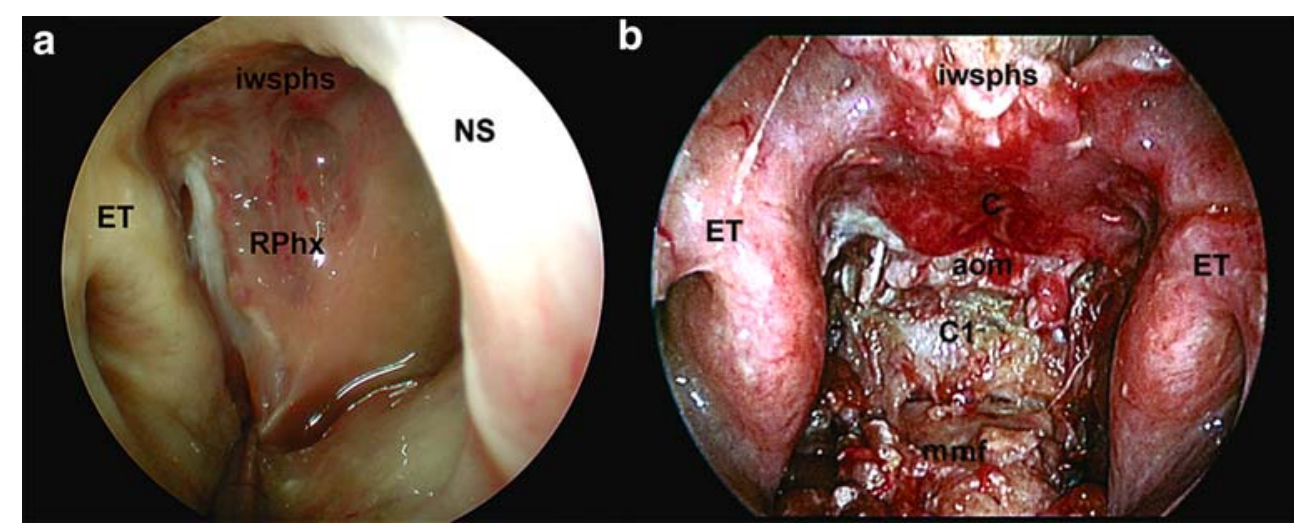




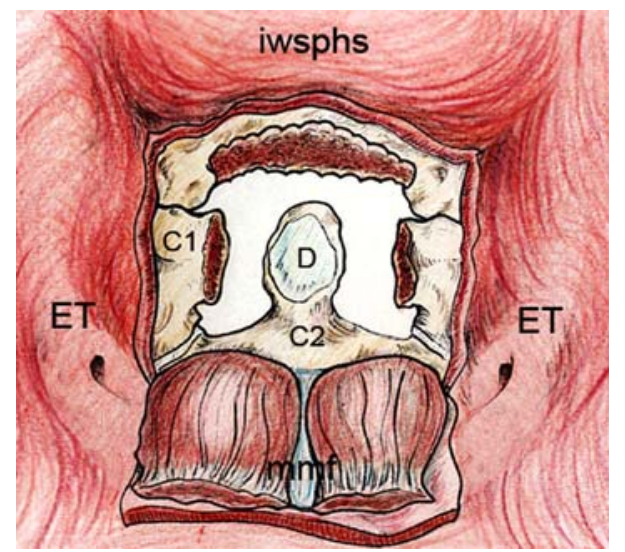

Fig. 3 Schematic drawing showing the muscle-mucosal flap and the lower structures (iwsphs inferior wall of sphenoid sinus, ET Eustachian tube, $C 1$ atlas, $m m f$ muscle-mucosal flap, $C 2$ axis, $D$ dens)

mucosa of the rhinopharinx, which provides a useful autologous material for closure of the surgical field.

Reintroducing the endoscope into the nasal cavity, the anterior arch of the atlas was removed and the dens with the apical and alar ligaments were exposed (see Fig. 5a,b). The dens was then thinned with a microdrill, separated from the alar and apical ligaments, and finally removed (see Fig. 6a,b).

At this point the transverse ligament was identified; it was removed with the tectorial membrane, a double layer membrane positioned behind the transverse ligament, in order to expose the dura mater. At the end of the procedure the muscle-mucosal flap was replaced into the nasal cavity, thus closing the surgical field (see Fig. 7).

In this study, the endoscopic endonasal approach to the cranio-vertebral junction has been performed using both the one-nostril and the two-nostril technique, without removal of inferior and/or middle turbinate, nasal septum or other nasal structures. Although the procedure can be performed through only one nostril, the binostril technique provides, without any additional surgical trauma, a better manoeuverability of the surgical tools and the possibility to work with "three hands". As a matter of fact, this technique permits a free-hand use of the endoscope in one nostril, held by the assistant, and the use of the other nostril or both nostrils for the insertion of the surgical instruments.

Furthermore, in the case of a narrow nasal cavity, it is valuable to perform a unilateral middle turbinectomy and removal of the posterior third of the nasal septum to enlarge the surgical corridor.

\section{Discussion}

Different pathological disorders may produce atlanto-axial translocation with ventral compression of the brain stem or spinal cord. The most common are congenital malformations, such as Arnold Chiari malformation type II, chronic inflammation, such as rheumatoid arthritis, genetic transformation, such as Down's syndrome and trauma, such as type II odontoid fracture. Some of these patients are candidate to the resection of the odontoid process for anterior decompression. The indication for odontoid resection is irreducible atlanto-axial subluxation, associated with severe spinal cord compression causing progressive myelopathy.

The most favoured approach to the odontoid process is the transoral approach. This approach provides a direct route to the surgical field, without any neurovascular manipulation and passing through the oropharynx, without injuring major neurovascular structures. The main limitation to this approach is the difficulty of dural closure and the subsequent higher risk of CSF leak and meningitis. For this reason the trans-oral approach is mainly used for extradural lesions [7, 8, 11-13, 15, 22-24], although some studies have reported its application for the surgical treatment of intradural pathology of the lower clivus and ventral cranio-cervical junction $[6,9,16,25,26]$. Other minor disadvantages are, however, related to this approach: the split of the soft palate and even of the hard palate is often performed in the case when rostral extension of the approach is required; tongue swelling may occur for prolonged compression; there is risk of damaging the teeth with retractors; velopharyngeal insufficiency may develop; and there is the necessity of nasal feeding in the postoperative stay.
Fig. 4 a The muscle-mucosal flap is transposed in the oropharinx. b Schematic drawing showing the exposure of the cranio-vertebral junction after replacing the flap. (Ophx oropharynx, $m m f$ musclemucosal flap, $T$ tongue)
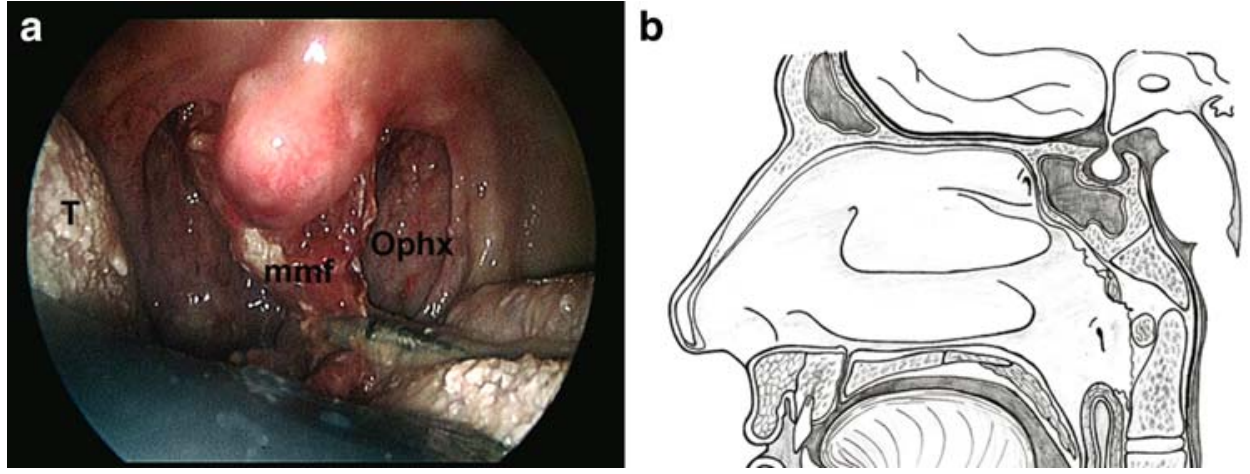
Fig. 5 a The anterior arch of the atlas has been removed. b The dens has been exposed. ( $C$ clivus, aom atlanto-occipital membrane, $C l$ atlas, $d m$ dura mater, al alar ligament, $D$ dens)

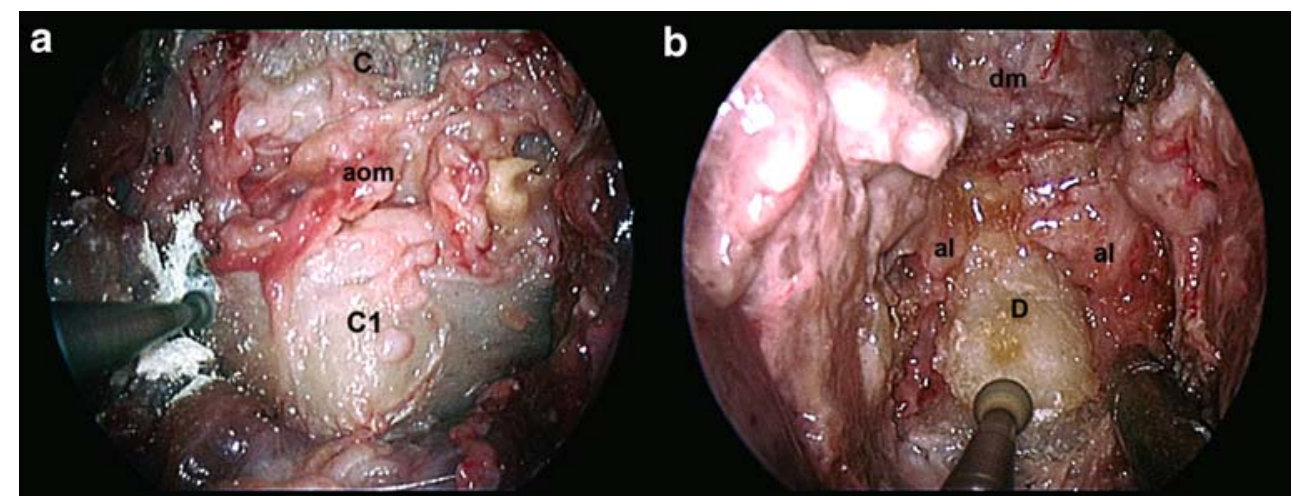

Recently, increased diffusion in the use of the endoscope for transsphenoidal pituitary surgery $[3,17]$ led some studies to explore the possibility of applying the endoscopic endonasal approach in the surgical treatment of skull base lesions other than pituitary tumors. In recent years some works have reported anatomical studies and surgical experience in the endoscopic endonasal approach to different areas of the midline skull base, from the olfactory groove to the cranio-vertebral junction $[1,2,4,5,10,14,18-21]$.

Thanks to the properties of the endoscope itself, the endonasal approach provides a wider view of the surgical field and a close-up vision, when compared with the transoral microscopic approach. Furthermore, the minimal invasiveness of the endoscopic endonasal route may reduce some morbidities related to the transoral approach. In fact, it is no longer necessary to use mouth retractors, prolonged compression of the tongue or split of the soft palate, and even considering the necessity of a middle turbinectomy or removal of the posterior portion of the nasal septum to enlarge the surgical corridor, these adjunctive manoeuvres do not usually produce morbidity to the patient. These manoeuvres are often performed in the endonasal extended approaches to the area around the sella in live patients and do not cause any respiratory problems.

The possibility of performing an odontoidectomy through the nose is strictly related to the level of the $\mathrm{C} 1-$ $\mathrm{C} 2$ junction. In fact, in the case of a low junction, below the level of the hard palate, it is virtually impossible to remove the odontoid process with an endonasal approach. On the contrary, in the case of a high position of the atlas-axis junction, the dens is more easily reached and removed through the nasal cavities.

Odontoidectomy may be considered one of the most complicated manoeuvres for the transoral approach, in which the split of the soft and even hard palate is often necessary. Thus, this approach could be evaluated for those cases in which a transoral removal is considered more difficult.

However, this kind of approach still presents some of the main problems of the transoral approach. The first problem concerns the risk of CSF leak and subsequent meningitis. Although the endoscope, thanks to its close-up and multi-angled vision, has a greater chance of detecting an occasional CSF leak, it is quite hard to suture the dura and the nasopharynx mucosa with conventional suturing tools through the nose. For this reason, in our anatomical study, we have created a muscle-mucosal flap, comprehensive of the entire muscular and mucosal tissue covering the ventral cranio-vertebral junction. This flap, as shown, is transposed into the oral cavity during the bone's removal and replaced in its original site at the end of the procedure. Due to the difficulty of anchoring the flap with suture, it is only distended on the defect and its borders are apposed on the corresponding lines of incision. The mucosa of the inferior wall of the sphenoid sinus could be stripped to favour the adherence of the flap and fibrin glue could be used to seal the edges. The

Fig. 6 a The dens has been drilled. b After removal of the odontoid process the transverse ligament and the underling tectorial membrane are visible. (iwsphs inferior wall of sphenoid sinus, ET Eustachian tube, $d m$ dura mater, $D$ dens)

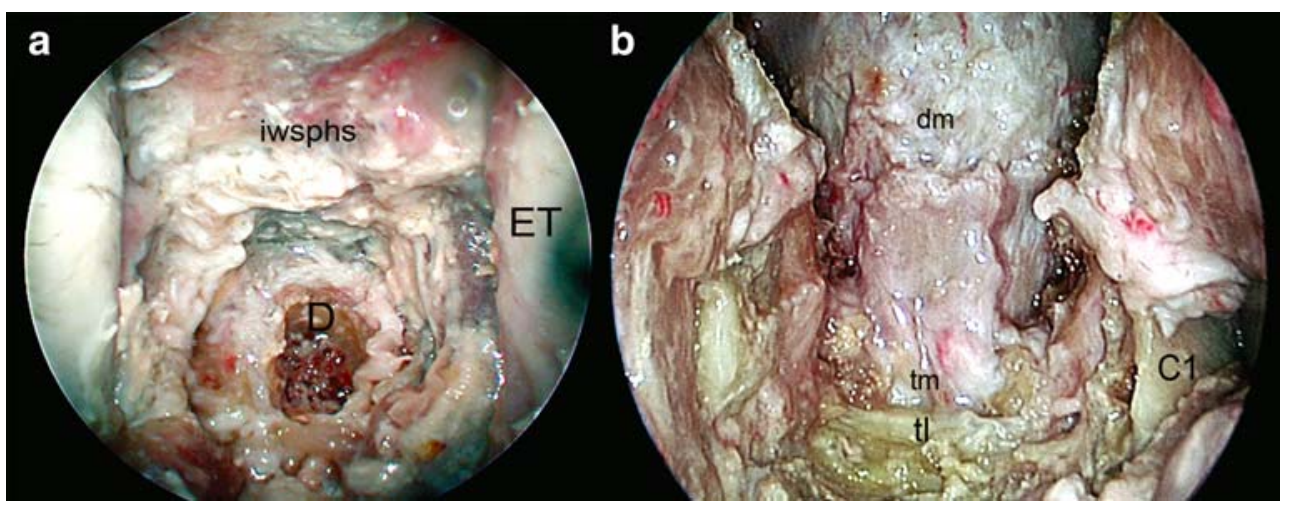




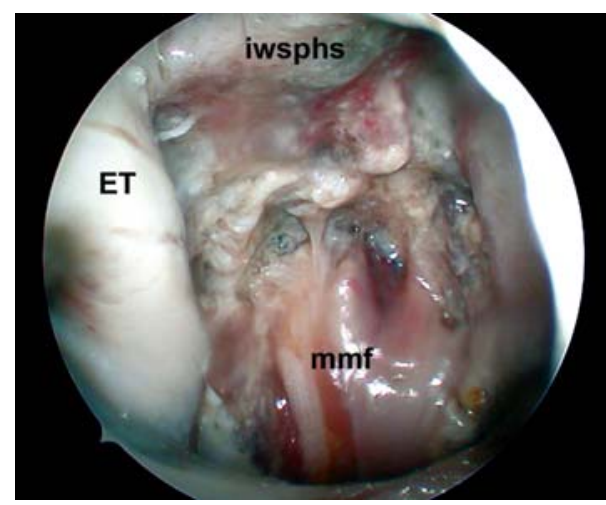

Fig. 7 The muscle-mucosal flap is replaced in the rhinopharinx (iwsphs inferior wall of sphenoid sinus, ET Eustachian tube, $\mathrm{mmf}$ muscle-mucosal flap)

creation of a peduncolated muscle-mucosal flap permits a more physiological reconstruction of the surgical corridor and, furthermore, the vascularization of the flap that directly continues with the oropharynx, facilitates a rapid healing. An endoscopic control should be performed one month after surgery to check the recreating integrity of the rhinopharynx mucosa.

The second problem of the transoral approach concerns the stability of the cranio-vertebral junction. The removal of the odontoid process with its ligaments can destabilize the cranio-vertebral junction.

The third problem concerns the haemostasis that is often difficult in the extended endonasal approaches. Bleeding control may become difficult with bipolar coagulation because the endonasal approach presents a long and narrow corridor, with a limited working space between the tips of the bipolar forceps. Nevertheless, specific bipolar forceps (TAKE-APART bipolar forceps; Karl Storz GmbH, Tuttlingen, Germany) have been used to work through the nose in a safe and effective way as well.

\section{Conclusions}

This cadaver study has been performed to demonstrate the possibility of an anterior decompression of the upper cervical cord through an endoscopic endonasal approach. Similar to the transoral approach, the endoscopic endonasal approach provides a direct route to the surgical target, but it seems related to less morbidity. For clinical applications of this approach, the most common surgical problems are the risk of CSF leak and meningitis, the instability of the cervico-medullary junction and the bleeding control. The creation of a muscle-mucosal flap may represent a valid modality for closure of the surgical field. For application in live surgery, dedicate surgical instruments, such as endonasal bipolar forceps, high-speed low-profile drill and surgical guidance systems are needed.
In selected cases this approach could be considered a valid alternative to the transoral microscopic approach for the resection of the odontoid process of $\mathrm{C} 2$. Obviously, it should be performed only by surgeons very skilled in endoscopic endonasal surgery and in endoscopic cadaverdissections, while cooperation in a team with ENT surgeons is recommended.

Acknowledgements The realization of this work and of all research of the "European Multicenter Anatomical Study for an innovative Endoscopic Endonasal Approach in Neurosurgery" was possible thanks to the grant I/05/A/PL-154419-SU of the Leonardo da Vinci European Community Vocational Training Action Programme (http://europa.eu.int/ comm/education/programmes/leonardo/leonardo_en.html).

\section{References}

1. Alfieri A, Jho HD, Schettino R, Tschabitscher M (2003) Endoscopic endonasal approach to the pterygopalatine fossa: anatomic study. Neurosurgery 52:374-380

2. Alfieri A, Jho HD, Tschabitscher M (2002) Endoscopic endonasal approach to the ventral cranio-cervical junction: anatomical study. Acta Neurochir 144:219-225

3. Cappabianca P, Alfieri A, de Divitiis E (1998) Endoscopic endonasal transsphenoidal approach to the sella: towards functional endoscopic pituitary surgery (FEPS). Minim Invas Neurosur 41:66-73

4. Cappabianca P, Frank G, Pasquini E, de Divitiis O, Calbucci F (2003) Extended endoscopic endonasal transsphenoidal approaches to the suprasellar region, planum sphenoidale and clivus. In: de Divitiis E, Cappabianca P (eds) Endoscopic endonasal transsphenoidal surgery. Springer, New York, pp 176-187

5. Cavallo LM, Messina A, Cappabianca P, Esposito F, de Divitiis E, Gardner P, Tschabitscher M(2005) Endoscopic endonasal surgery of the midline skull base: anatomical study and clinical considerations. Neurosurg Focus 19:E2

6. Crockard HA (1993) Transoral approach to intra/extradural tumors. In: Sekhar LN, Janecka I (eds) Surgery of cranial base tumors. Raven Press, New York, pp 225-234

7. Crockard HA, Bradford R (1985) Transoral transclival removal of a schwannoma anterior to the craniocervical junction. Case report. J Neurosurg 62:293-295

8. Crockard HA, Pozo JL, Ransford AO, Stevens JM, Kendall BE, Essigman WK (1986) Transoral decompression and posterior fusion for rheumatoid atlanto-axial subluxation. J Bone Jt Surg Br 68:350-356

9. Crockard HA, Sen CN (1991) The transoral approach for the management of intradural lesions at the craniovertebral junction: Review of 7 cases. Neurosurgery 28:88-98

10. de Divitiis E, Cappabianca P, Cavallo LM (2002) Endoscopic transsphenoidal approach: adaptability of the procedure to different sellar lesions. Neurosurgery 51:699-707

11. Dickman CA, Spetzler RF, Sonntag VK (1998) Surgery of the craniovertebral junction. Thieme, New York

12. Di Lorenzo N (1992) Craniocervical junction malformation treated by transoral approach. A survey of 25 cases with emphasis on postoperative instability and outcome. Acta Neurochir 118:112-116

13. Donald PJ (1998) Transoral approach to the clivus and upper cervical spine. In: Donald PJ (ed) Surgery of the skull base. Lippincott-Raven, Philadelphia, pp 507-532

14. Frank G, Pasquini E, Mazzatenta D (2001) Extended transsphenoidal approach. J Neurosurg 95:917-918 
15. Hadley MN, Spetzler RF, Sonntag VK (1989) The transoral approach to the superior cervical spine. A review of 53 cases of extradural cervicomedullary compression. J Neurosurg 71:16-23

16. Hayakawa T, Kamikawa K, Ohnishi T, Yoshimine T (1981) Prevention of postoperative complications after a transoral transclival approach to basilar aneurysms. Technical note. J Neurosurg 54:699-703

17. Jho HD, Carrau RL, Ko Y (1996) Endoscopic pituitary surgery. In: Wilkins H, Rengachary S (eds) Neurosurgical operative atlas. American Association of Neurological Surgeons, Park Ridge, pp $1-12$

18. Jho HD, Carrau RL, McLaughlin MR, Somaza SC (1997) Endoscopic transsphenoidal resection of a large chordoma in the posterior fossa. Acta Neurochir 139:343-348

19. Jho HD, Ha HG (2004) Endoscopic endonasal skull base surgery: Part 3. The clivus and posterior fossa. Minim Invas Neurosur 47:16-23

20. Kassam A, Snyderman CH, Mintz A, Gardner P, Carrau RL (2005) Expanded endonasal approach: the rostrocaudal axis. Part II. Posterior clinoids to the foramen magnum. Neurosurg Focus 19:E4

21. Kassam AB, Snyderman C, Gardner P, Carrau R, Spiro R (2005) The expanded endonasal approach: a fully endoscopic transnasal approach and resection of the odontoid process: technical case report. Neurosurgery 57:E213

22. Menezes AH, Graf CJ, Hibri N (1980) Abnormalities of the craniovertebral junction with cervico-medullary compression. A rational approach to surgical treatment in children. Childs Brain 7:15-30

23. Menezes AH, VanGilder JC (1988) Transoral-transpharyngeal approach to the anterior craniocervical junction. Ten-year experience with 72 patients. J Neurosurg 69:895-903

24. Menezes AH, VanGilder JC, Clark CR, el-Khoury G (1985) Odontoid upward migration in rheumatoid arthritis. An analysis of 45 patients with "cranial settling". J Neurosurg 63:500-509
25. Miller E, Crockard HA (1987) Transoral transclival removal of anteriorly placed meningiomas at the foramen magnum. Neurosurgery 20:966-968

26. Reisch R, Bettag M, Perneczky A (2001) Transoral transclival removal of anteriorly placed cavernous malformations of the brainstem. Surg Neurol 56:106-116

\section{Comments}

\section{Martin Bettag, Trier, Germany}

The authors provide an anatomical study of endoscopic removal of the odontoid process as an alternative to the otherwise most favoured microsurgical transoral approach. They nicely demonstrate this midline approach with respect to the surrounding structures. Creating a muscle-mucosal flap seems to be a good solution for the closure of the surgical field.

However, some details have to be discussed. At first, the endonasal approach to the craniovertebral junction offers only a long and narrow surgical corridor so that even using both nostrils the insertion and the use of instruments can be very difficult. Especially the use of standard needle holders or bipolar forceps is almost impossible. Also, with the endonasal technique, one can only approach the $\mathrm{C} 1-\mathrm{C} 2$ level if this level is not located below the level of the hard palate. In the case of a low junction, the endonasal approach is not feasible. On the contrary, the transoral approach to the craniovertebral junction permits a wide access from the inferior third of the clivus to the $\mathrm{C} 3$ vertebra.

In conclusion, this is a well written report by recognized leaders in the field of endoscopic endonasal surgery. The given anatomical details and technical solutions are very precise and instructive. However, I doubt that there are many reasonable indications in patients where an endoscopic endonasal approach will be superior to the standard transoral odontoidectomy. 